Brajković, M., Barac, M., Cosic, D., Bogdanović Radović, I. \& Siketić, Z. (2019) Development of MeV TOF-SIMS capillary microprobe at the Ruđer Bošković Institute in Zagreb. Nuclear instruments \& methods in physics research. Section B, Beam interactions with materials and atoms, 461, 237-242 doi:10.1016/j.nimb.2019.10.006.

Accepted Manuscript

\title{
Development of MeV TOF-SIMS capillary microprobe at the Ruđer Bošković Institute in Zagreb
}

\author{
Marko Brajković ${ }^{1}$, Marko Barac ${ }^{1,2}$, Donny Cosic $^{1}$, Iva Bogdanović Radović, Zdravko Siketić ${ }^{*}$ \\ ${ }^{1}$ Laboratory for Ion Beam Interactions, Department of Experimental Physics, Ruđer Bošković Institute, \\ Bijenička c. 54, 10000 Zagreb, Croatia

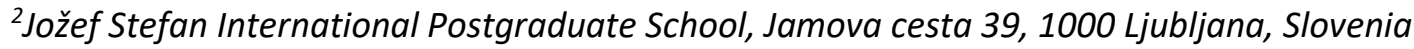

\begin{abstract}
New Time-of-flight Secondary lon Mass Spectrometry (TOF SIMS) setup using MeV heavy ions for the excitation is developed at the Ruđer Bošković Institute accelerator facility. To focus heavy MeV ions to micron dimensions, conical glass capillary is used instead of quadrupole magnetic lenses. The setup uses a continuous primary beam where START signal for TOF is obtained from the PIN diode placed behind the thin transmission sample. Measured energy spectra for several primary heavy ions are presented and compared with theoretical simulations. The first mass spectra obtained with the new setup using reflectron-type TOF analyser are given together with the mass and spatial resolution values of the new setup.
\end{abstract}

Keywords: MeV TOF-SIMS, capillary microprobe, biomolecular imaging

*Corresponding author: zsiketic@irb.hr

\section{Introduction}

To overcome high fragmentation of large molecules and consequently complex mass spectra obtained by conventional (keV) Time-of-flight Secondary lon Mass Spectrometry (TOF-SIMS), in the last couple of years several setups were designed at the accelerator facilities worldwide which utilize focused MeV heavy ions for the excitation $[1,2,3,4,5]$. Since the electronic stopping is the main energy loss mechanism for interaction between MeV heavy ions and the sample surface, molecules are more softly desorbed and therefore up to several orders of magnitude higher yields of intact molecules are expected than in case when keV ions are used [6]. Therefore, MeV TOF-SIMS spectra are in general easier to analyse comparing to their low energy counterpart, which makes this method a useful tool in molecular imaging and surface characterization of organic samples $[7,8,9]$. The major limiting factor of this technique is inability of proper focusing of high energy heavy ions which are bringing the highest benefit through their large electronic stopping power values with the existing standard magnetic quadrupole lenses. One way to overcome this problem is to use alternative setup: instead of using ion optical lens system, one can use capillary microprobe system where the primary beam is collimated by passing through the small opening of glass capillary $[5,10,11]$. The target beam current in that case is not merely defined by ratio of input and output radii of the capillary, but also depends on the capillary shape and material. Interaction of ions with capillary walls through Rutherford scattering results in an enhancement of output beam current [12]. 
In present paper, progress in the development of the capillary microprobe system recently installed at the Ruđer Bošković Institute accelerator facility is presented. The new chamber, coupled to reflectron timeof-flight (TOF) analyser (manufactured by Kore Technology Ltd), is an upgrade of the existing MeV TOFSIMS setup with quadrupole triplet focusing and linear TOF analyser installed at the heavy ion nuclear microprobe beamline. The old setup (described in detail Ref. [3]) is limited to focus ions with rigidity less than 14 (equivalent to $8 \mathrm{MeV} \mathrm{Si}^{4+}$ ), therefore not allowing use of heavier and more energetic ions such as $23 \mathrm{MeV} \mathrm{I}^{6+}$ ions which are standardly used for TOF Elastic Recoil Detection Analysis (TOF-ERDA) measurements at $0^{\circ}$ beam line. Heavy ions can be provided either by 6 MV HVEC Tandem Van de Graff accelerator or by 1 MV HVEE Tandetron accelerator. Therefore, new setup that is using capillary for obtaining micrometre beam dimension is built in the extension of the existing TOF-ERDA beam line which can accept ions from both accelerators, with no restriction on the ion rigidity. Since the beam current is heavily reduced by passing through the capillary, pulsed beam which is used for triggering of START signal at the old MeV TOF SIMS setup at the heavy ion microprobe with magnetic focusing is no longer an option. It would take too long to obtain enough statistics in the spectrum using this trigger method, so new triggering options needed to be considered. For thin targets, as a START trigger, particle detector in transmission geometry is always an option. Major disadvantage of this option is radiation damage suffered by such a detector due to the fact that beam is not scanned over the sample surface. Instead, the beam is fixed so charge collection efficiency of the particle detector reduces over time leading to a relatively short detector's lifetime. Consequently, one needs to frequently replace the detector with the new one in order to have a reliable START signal. As presently, our capillary microprobe system is equipped only with this detector, so MeV TOF-SIMS setup was tested using thin transmission targets. Installation of an electron detector, which will detect secondary electrons created after interaction of ions with the target and provide START signal for TOF measurements regardless of sample thickness, is in progress.

\section{Experimental Setup}

Schematic view of the new chamber is shown in Fig. 1. The system is operating in high vacuum of $\sim 10^{-7}$ $\mathrm{hPa}$. A sample holder is placed about $40 \mathrm{~mm}$ from the capillary outlet at $45^{\circ}$ to the incident beam direction. Grounded extractor tip is placed perpendicular to the sample surface at the distance of $5 \mathrm{~mm}$. Additionally, during the measurements sample inclination can be slightly varied $\left( \pm 1^{\circ}\right)$ in order to maximize the secondary molecular ion yield. By applying up to $5 \mathrm{kV}$ to the sample $\left(\mathrm{V}_{\text {target }}\right)$, the electric field is created between the sample surface and extractor tip which enables extraction of secondary molecular ions from the sample. After entering the TOF analyser and passing through $586 \mathrm{~mm}$ field-free region, ions are reflected in the electric field which can be controlled through Reflect $\left(V_{\text {reflect }}\right)$ and Retard $\left(V_{\text {retard }}\right)$ voltages of the reflectron-type analyser. The reflected ions travel again through $296 \mathrm{~mm}$ of field-free region and enter $25 \mathrm{~mm}$ diameter dual micro channel plate (DMCP) detector with flange-mounted external fast preamplifier. Mass resolution of the analyser can be optimized by adjusting Reflect and Retard voltages, as well as Einzel lens voltage $\left(V_{\text {Einzel }}\right)$ placed after the entrance of the extractor. The best obtained time resolution for the $\mathrm{H}$ ion $(\mathrm{m} / \mathrm{q}=1)$ was $5 \mathrm{~ns}$ with following values for the voltages: $\mathrm{V}_{\text {target }}=4.5 \mathrm{kV}, \mathrm{V}_{\text {reflect }}=$ $4.6 \mathrm{kV}, \mathrm{V}_{\text {retard }}=3.1 \mathrm{kV}, \mathrm{V}_{\text {Einzel }}=2.4 \mathrm{kV}$ and $\mathrm{V}_{\mathrm{MCP} \text {-front }}=-2 \mathrm{kV}$. A $5 \times 5 \mathrm{~mm}^{2}$ PIN diode, which triggers the TOF START signal, is placed around $10 \mathrm{~mm}$ behind the target and negatively biased (100-200 V) through Ortec $142 \mathrm{~A}$, charge sensitive preamplifier. Both START (timing signal from charge sensitive preamplifier) and STOP (pre-amplified MCP signal) signals go through same electronic chain: first to Constant Fraction Discriminators (CFDs), followed by Timing filter amplifiers which are then connected to the homemade Multistop Time to Digital Converter data acquisition system SPECTOR v2 [13]. Coincidence window can 
be set in SPECTOR v2 which controls the acquisition and displays acquired spectra (usually, $100 \mu \mathrm{s}$ acquisition window over $32 \mathrm{k}$ channels is used).

The sample holder is mounted on a 4D piezo-based scanning stage (producer SmartAct) which enables measurement of spatial distribution of molecules (molecular imaging). Optimum speed of the scanning stage is $100 \mu \mathrm{m} / \mathrm{s}$, and nominal lateral resolution of each piezo stage is $1 \mathrm{~nm}$. Pre-set time or collected charge in the PIN diode (normalization to number of counts in the diode) can be used as a trigger to move stage to the new position. During moving to the new position, spectrum is not acquired.

\section{Results}

\section{Energy spectra of the primary ion beam transmitted through the capillary}

The capillary, produced at the ETH Zürich, is mounted on a precision tilt port aligner enabling initial aligning of capillary with the beam direction. It is made out of borosilicate glass and conically shaped, with inlet diameter of $0.86 \mathrm{~mm}$ and nominal outlet diameter of $\sim 5 \mu \mathrm{m}$. When ion enters the capillary it can either directly pass through it without interacting with the capillary walls (probability of this event is determined by the square of the inlet to outlet diameter ratio) or it can collide with the capillary walls through Rutherford (low-angle) scattering, with several different end-results: i) ion can lose all energy inside the capillary walls, ii) it can be backscattered inside the capillary iii) it can be forward scattered exiting the capillary or iv) it can pass through the capillary walls at the capillary tip losing some energy inside the thin capillary walls. Following procedure outlined by M.J. Simon et al. described in Ref. [12] (SRIM [14] calculated trajectories of primary beam ions inside borosilicate glass capillary) simulations of energy spectra and spatial distribution of ions in the detector were performed and are shown in Fig. 2. For the simulations it was assumed that ion beam is perfectly aligned with the capillary axis.

Experimentally obtained energy spectra for two different energies of Au ions and three different energies of $\mathrm{I}$ ions after passing through the glass capillary are shown in Figs. $3 a$ and $3 b$, respectively. Figure $3 c$ shows spectra collected in the PIN diode for several different ions (I, $\mathrm{Cu}, \mathrm{Au}, \mathrm{Si}$ ) with similar incident energy. The ions passing directly though the capillary are detected with the full energy peak, while low energy tail can be associated to the ions scattered at the capillary walls as well as transmitted through the capillary tip. Simulations show that contribution from the ions transmitted through the walls is a minor more than $99 \%$ of ions in low energy tail come from scattering at the capillary walls. Simulations for $\mathrm{Au}$ ions with different energies (Figs. $2 b$ and $2 c$ ) show that low energy tail is always present, but its contribution depends on ions' range inside the glass capillary (ions with lower energy have higher chance of losing all energy inside the capillary walls). Also, low energy resolution of the used particle detector plays the role - low energy tail is sometimes "hidden" inside the broad full energy peak in measured spectra (Figs. 3a-3c). Since the capillary walls are very thin at the capillary end, small variation in ion range can significantly influence energy spectra of transmitted ions, which is shown in Fig. 2c where much longer low-energy tail for $15 \mathrm{MeV} \mathrm{Si}$ ion can be seen compared to the $17.5 \mathrm{MeV} \mathrm{Au}$ ion in Fig. 2b, although difference in the range calculated by SRIM is only $1.6 \mu \mathrm{m}$. This is also shown in experimental spectrum in Fig. $3 c$ so one has to have it in mind when considering the optimum ion for the measurement. Influence of the energy distribution on the time resolution can be easily calculated (ions with lower energy need more time to travel from the target to the diode placed at the distance of $10 \mathrm{~mm}$ behind the target). If we assume for example that energy range of $1 \mathrm{MeV}$ covers central peak and large majority of low-energy tail for $17.5 \mathrm{MeV} \mathrm{Au}$ in Fig. 2b, one can roughly estimate that the time spread is $\sim 70 \mathrm{ps}$. In the worst case, 
for $15 \mathrm{MeV} \mathrm{Si}$ (due to the long low-energy tail), this contribution goes up to few hundreds of ps which is negligible comparing to the measured intrinsic time resolution of $5 \mathrm{~ns}$.

Simulated spatial distribution of the ions (Fig. 2d) shows that scattered ions create halo around directly transmitted ions, which is expected given the conical shape of the capillary which determines minimal exit angle for the scattered ions. That was confirmed experimentally using Gafchromic radiosensitive EBT3 film placed on the sample holder and exposed to the $5 \mathrm{MeV} \mathrm{Si}$ ion beam (Fig. 4). Deformed shape of halo is a result of non-perfect alignment of ion beam with capillary axis [12] and $45^{\circ}$ angle between incident beam direction and sample surface.

Spatial distribution of molecules is created by collection of spectra in each pixel which size depends on the beam spot size. When central part of the beam is located in one pixel, ions from the halo will hit the sample around this pixel creating "false" counts in this pixel's spectrum. In that way, existence of halo will blur spatial distribution of molecules in each pixel which brings certain challenge for imaging applications that should be somehow solved in the future. Simulations also predict enhancement factor defined as ratio between number of all ions in the full energy peak (directly transmitted and scattered) to the number of ions directly transmitted in the full energy peak. Simulations predict this factor to be in range 1.4-1.6, depending on ion type and energy. In real measurement this enhancement factor can be even larger due to the capillary surface roughness and neglected plural scattering contribution in the simulations [12].

\section{Mass and spatial resolution}

First MeV TOF-SIMS spectra measured using $8 \mathrm{MeV} \mathrm{Si}^{4+}$ beam on thin Phthalocyanine blue (PB15:1, $\mathrm{C}_{32} \mathrm{H}_{16} \mathrm{CuN}_{8}$, m/q $\left.576.1 \mathrm{Da}\right)$ and leucine $\left(\mathrm{C}_{6} \mathrm{H}_{13} \mathrm{NO}_{2}, \mathrm{~m} / \mathrm{q} 131.2 \mathrm{Da}\right)$ layers evaporated on $1.5 \times 1.5 \mathrm{~mm}^{2}$ $100 \mathrm{~nm}$ thick $\mathrm{Si}_{3} \mathrm{~N}_{4}$ windows are shown in Figs. 5 and 6, respectively. Beam frequency was around $5 \mathrm{kHz}$ in the PIN diode, while the MCP counting rate was about $10 \%$ of that value. Best achieved time resolution for $\mathrm{m} / \mathrm{q}$ equal to 1 was $5 \mathrm{~ns}$. Measured mass resolution (defined as ratio between centroid of the peak and its full width at half maximum) for selected mass peaks are shown in Table 1 . Resolution of almost 2500 was achieved for group of peaks with $\mathrm{m} / \mathrm{q}$ around $576 \mathrm{Da}$, which is $3-5$ times better compared to the existing setup [3]. Background in the spectra was caused by random coincidences with halo ions molecular ions are sputtered from the sample and detected in the MCP but START signal is not detected so new ion can trigger TOF measurement creating false coincidence event in the mass spectrum.

Fig 7. represents distribution of counts in SIMS spectrum when $5 \mathrm{MeV} \mathrm{Si}^{4+}$ beam was scanned $(250 \times 5$ $\mu \mathrm{m}^{2}$ ) over the sharp edge (better than $100 \mathrm{~nm}$ ) of leucine evaporated on $\mathrm{Si}_{3} \mathrm{~N}_{4}$ window. The beam current at the capillary inlet was used as a normalization for the movement of the stage during the scanning. When primary beam hits the sample completely outside the window (minimum number of events), signals come only from the random coincidences from halo ions (left flat part of the distribution). Right-hand side of the distribution corresponds to core of the primary beam hitting the sample inside the window. Measured resolution in $\mathrm{x}$ direction, defined as the distance required for the number of counts to rise from $10 \%$ to $90 \%$ percent of the gap between minimum and maximum values of fit curve, is $22 \pm 1 \mu \mathrm{m}$. The same result is measured for resolution in y-direction ( $5 \times 250 \mu \mathrm{m}^{2}$ line scan). Two main factors contribute to worse than expected lateral resolution for this capillary: first is primary beam halo and second not sufficiently good ion beam optics of the experimental beam line. The latter results in minimum primary beam divergence of $\sim 0.04^{\circ}$ ( $\sim 1 \mathrm{~mm}$ collimator opening at $1.5 \mathrm{~m}$ before the capillary) which is significant if we take into account that the distance between the capillary tip and the sample is $40 \mathrm{~mm}$. 


\section{Conclusions}

We presented first results obtained from a newly developed MeV TOF-SIMS setup based on the microcapillary focusing system. A limiting factor, focusing power of the magnetic optical elements (ion rigidity) is eliminated and full capability of the MeV TOF-SIMS using heavy ions with high electronic stopping power can be utilized. Measured energy distribution of the transmitted ions through the capillary is in good agreement with the performed simulations and it does not significantly affect timing/mass resolution of the setup when PIN diode is used as a trigger for the START signal for TOF measurements (it contributes less than $1 \mathrm{~ns}$ to timing resolution of DAQ system). Mass resolution of the new setup based on reflectrontype TOF analyser is increased for a factor of 3 - 5 comparing to the simple linear TOF analyser used in the old setup. In this sense, accuracy of the mass peak identification, as well as sensitivity, is highly improved. Measured spatial resolution is $22 \mu \mathrm{m}$ for both $\mathrm{x}$ and $\mathrm{y}$ direction. That will be improved by using a capillary with a smaller outlet diameter as well as by optimizing the ion beam optics of the experimental line (new collimating slits will be installed prior to the chamber to minimize primary beam divergence). Next step in the development of the MeV TOF-SIMS microprobe will be design of a new triggering system for the START of TOF measurements based on the (secondary) electrons coming from the primary beam-target interaction, which will enable TOF-SIMS measurements for both thin and thick samples.

\section{Acknowledgements}

M. Brajković acknowledges support by Croatian Science Foundation (CSF) project "Young Researchers' Career Development Project - Training of Doctoral Students" co-financed by the European Union, Operational Program "Efficient Human Resources 2014-2020" and the ESF. I. B. R. and Z. S. acknowledge support by CSF project IP-2016-06-1698 "Development of a capillary microprobe for MeV SIMS for analysis of biological materials- BioCapSIMS" and RADIATE project under the Grant Agreement 824096 from the EU Research and Innovation programme HORIZON 2020.

Authors also wish to thank Dr. Max Döbeli and Dr. Klaus-Ulrich Miltenberger from ETH Zürich for the help with micro-capillary production; and Andro Kovačić for fabricating different parts of the system in the laboratory workshop.

\section{References}

1. Y. Nakata, Y. Honda, S. Ninomiya, T. Seki, T. Aoki, J. Matsuo, J. Mass. Spectrom. 44 (2009) 128136

2. B. N. Jones, V. Palitsin, R. Webb, Nucl. Instr. Meth B 268 (2010) 1714-1717

3. T. Tadić, I. Bogdanović Radović, Z. Siketić, D. D Cosic, N. Skukan, M. Jaksic, J. Matsuo, Nucl. Instr. Meth B 332 (2014) 234-237

4. L. Jeromel, Z. Siketić, N. Ogrinc Potočnik, P. Vavpetič, Z. Rupnik, K. Bučar, P. Pelicon, Nucl. Instr. Meth B 332 (2014) 22-27

5. M. Schulte-Borchers, M. Döbeli, A. M. Müller, M. George, H.-A. Synal, Nucl. Instr. Meth B 380 (2016) 94-98

6. I. Kamensky, P. Håkansson, B. Sundqvist, Nucl. Instr. Meth 198 (1982) 65-68

7. J. Matsuo, S. Ninomiya, H. Yamada, K. Ichiki, Y. Wakamatsu, M. Hada, T. Seki, T. Aoki, Surf. Interface Anal. 42 (2010) 1612-1615

8. B. N. Jones, J. Matsuo, Y. Nakata, H. Yamada, J. Watts, S. Hinder, V. Palitsin, R. Webb, Surf. Interface Anal. 43 (2011) 249-252 
9. M. J. Bailey, B. N. Jones, S. Hinder, J. Watts, S. Bleay, R.P. Webb, Nucl. Instr. Meth. B 268 (2010) 1929-1932

10. K.-U. Miltenberger, M. Schulte-Borchers, M. Döbeli, A. M. Müller, M. George, H.-A. Synal, Nucl. Instr. Meth B 412 (2017) 185-189

11. T. Ikeda, Y. Kanai, Y. Iwai, T. M. Kojima, K. Maeshima, W. Meissl, T. Kobayashi, T. Nebiki, S. Miyamoto, G. P. Pokhil, T. Narusawa, N. Imamoto, Y. Yamazaki, Surface \& Coatings Technology 206 (2011) 859-863

12. M.J. Simon, C.L. Zhou, M. Döbeli, A. Cassimi, I. Monnet, A. Méry, C. Grygiel, S. Guillous, T. Madi, A. Benyagoub, H. Lebius, A.M. Müller, H. Shiromaru, H.-A. Synal, Nucl. Instr. Meth B 330 (2014) 11-17

13. D. Cosic, M. Bogovac, M. Jakšić, Nucl. Instr. Meth B 451 (2019) 122-126

14. J. F. Ziegler, M. D. Ziegler, J. P. Biersack, Nucl. Instr. Meth B 268 (2010) 1818-1823

\section{Figures}

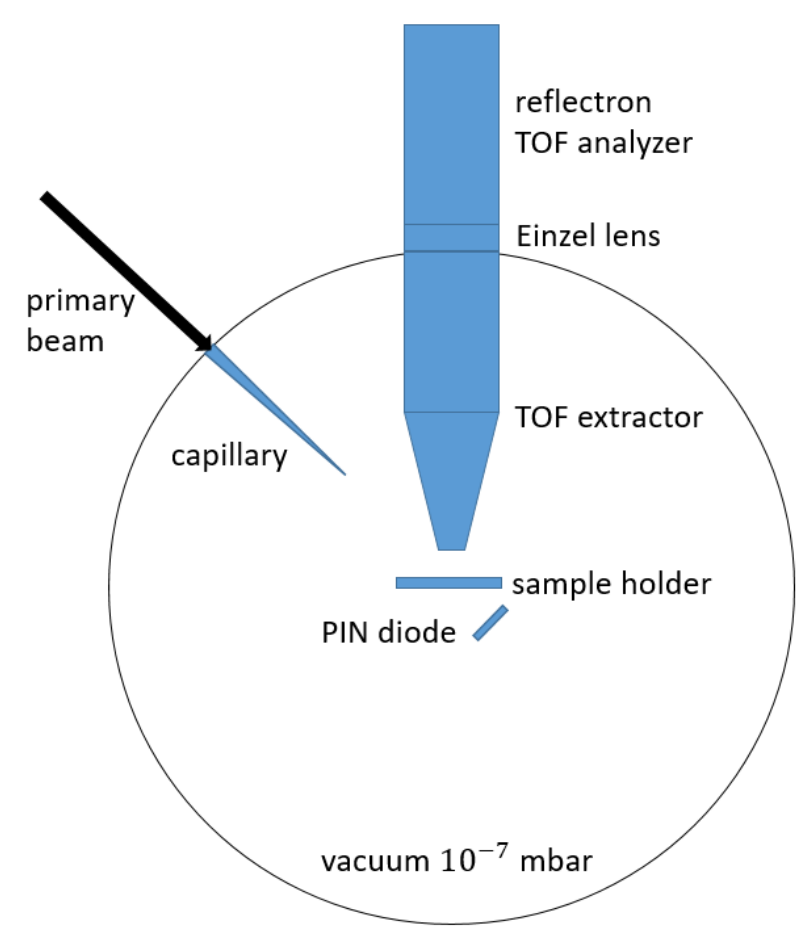

Figure 1 Schematic view of MeV SIMS capillary microprobe setup 


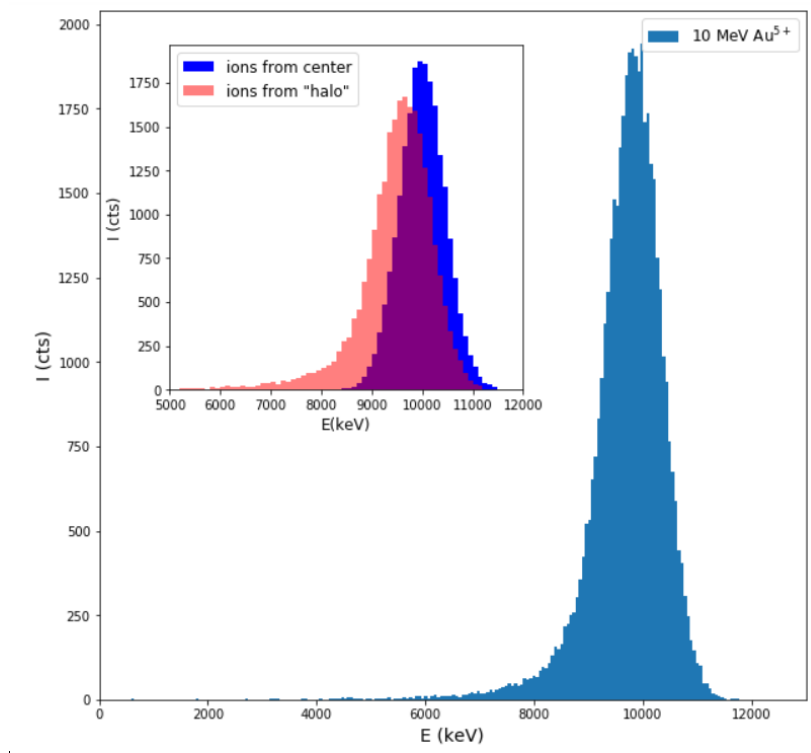

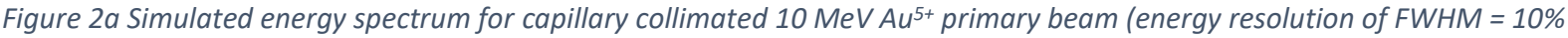
assumed). Low-energy tail is caused by scattered ions which form halo around the central part of the beam.

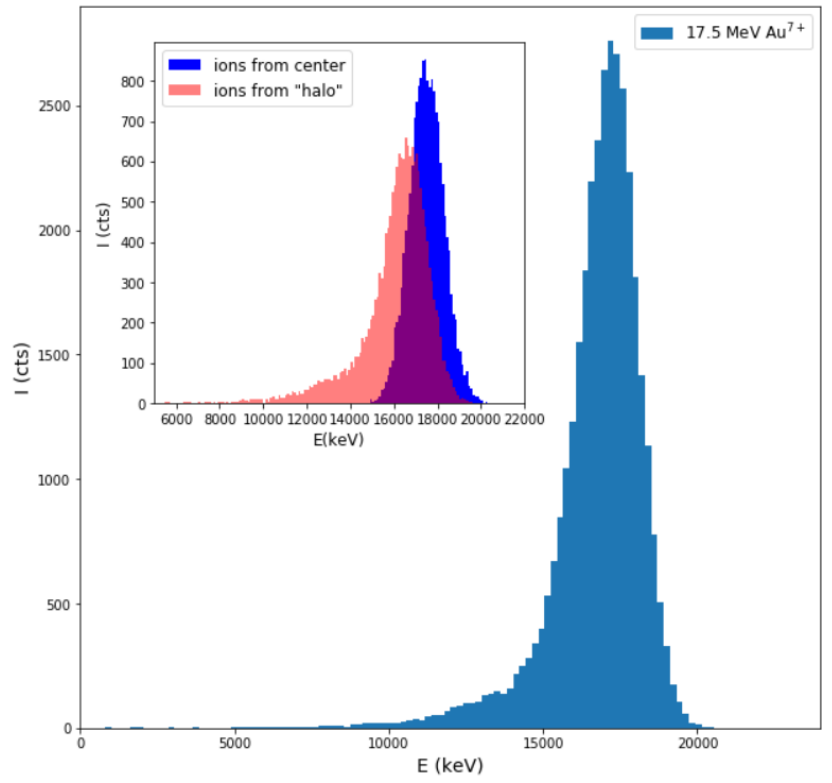

Figure $2 b$ Simulated energy spectrum for capillary collimated $17.5 \mathrm{MeV} \mathrm{Au}^{7+}$ primary beam (energy resolution of FWHM $=10 \%$ assumed). Low-energy tail is caused by scattered ions which form halo around the central part of the beam. 


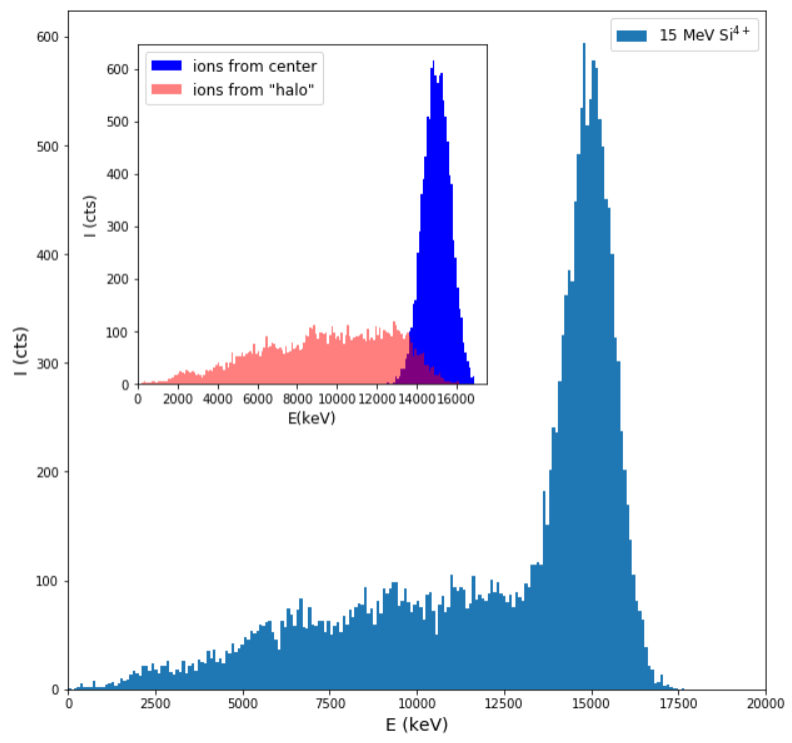

Figure 2c Simulated energy spectrum for capillary collimated $15 \mathrm{MeV} \mathrm{Si}^{4+}$ primary beam (energy resolution of FWHM $=10 \%$ assumed). Low-energy tail is caused by scattered ions which form halo around the central part of the beam

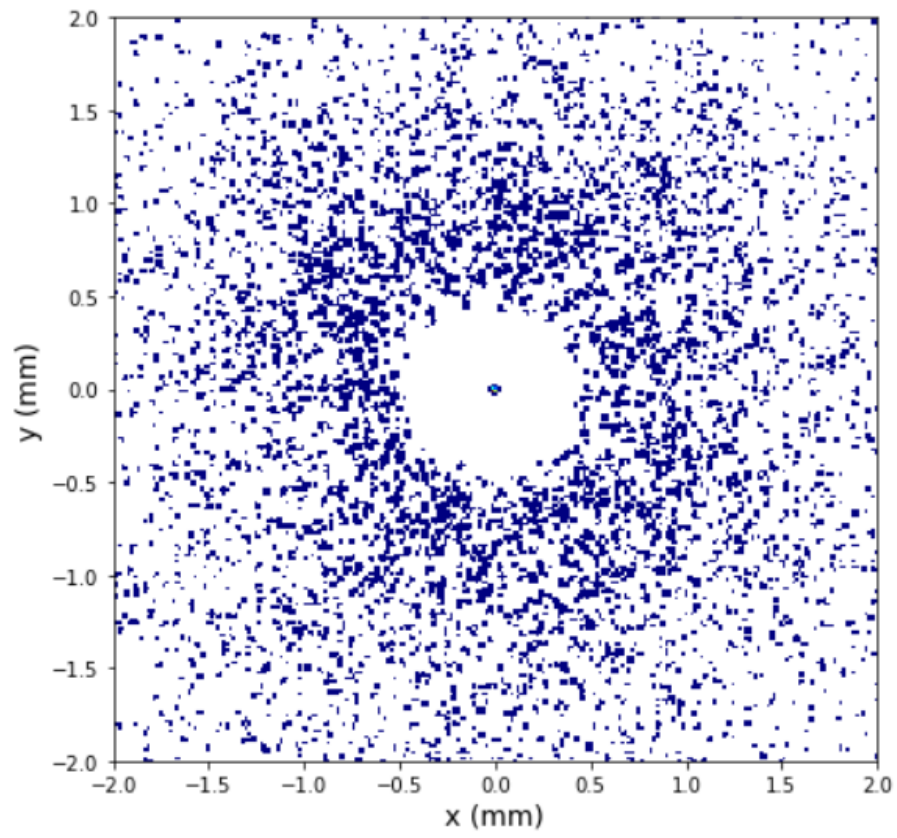

Figure $2 d$ Simulated spatial distribution of the capillary-collimated $5 \mathrm{Mev} \mathrm{Si}{ }^{4+}$ primary beam at the target. 


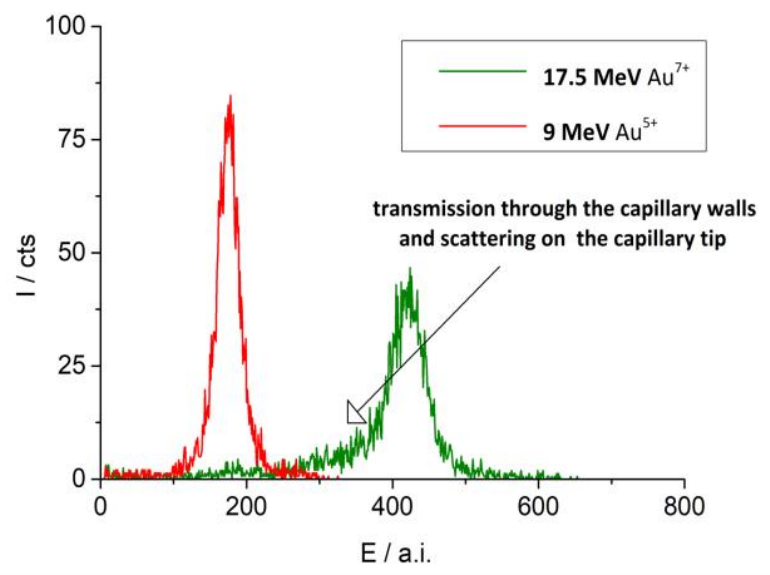

Figure 3a Energy spectra of Au beam for two different energies

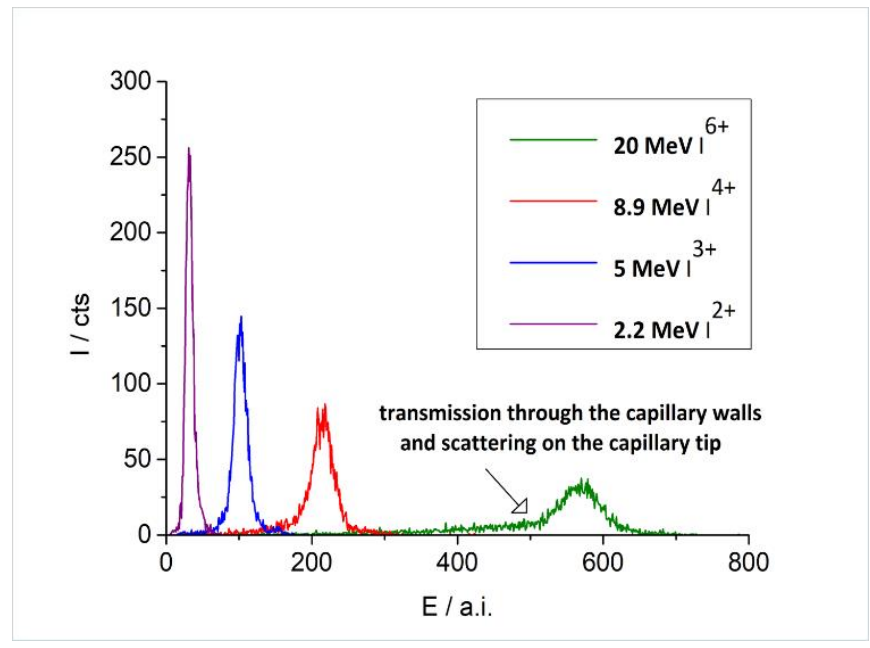

Figure $3 b$ Energy spectra of I beam transmitted through the capillary for different beam energies

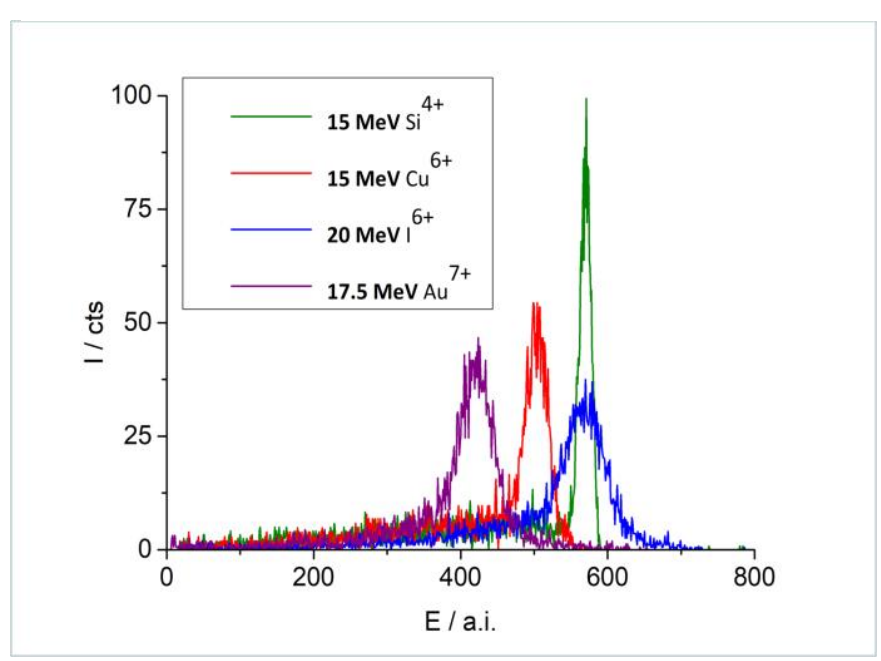

Figure 3c Energy spectra of different ions transmitted through the capillary 


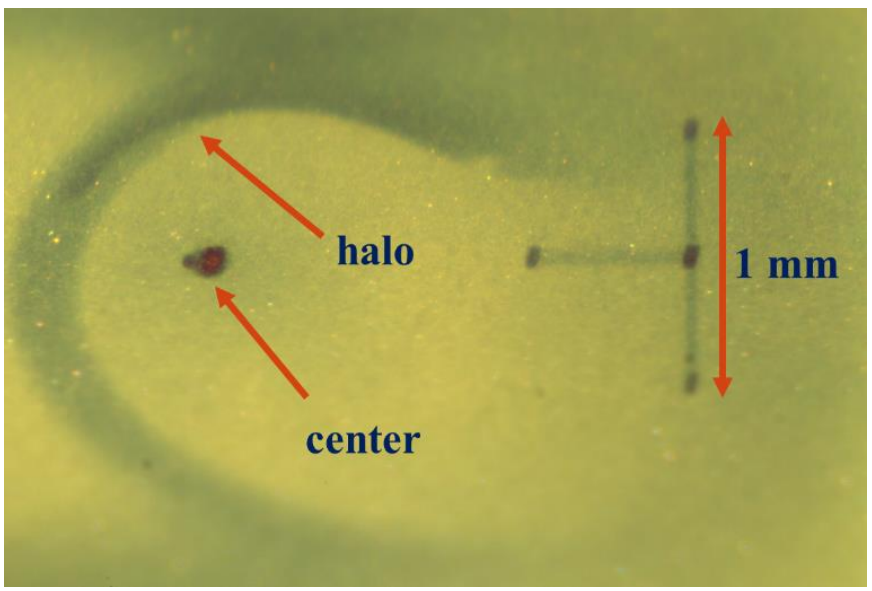

Figure 4 Gafchromic radiosensitive EBT3 film exposed to capillary focused $5 \mathrm{MeV} \mathrm{Si}{ }^{4+}$

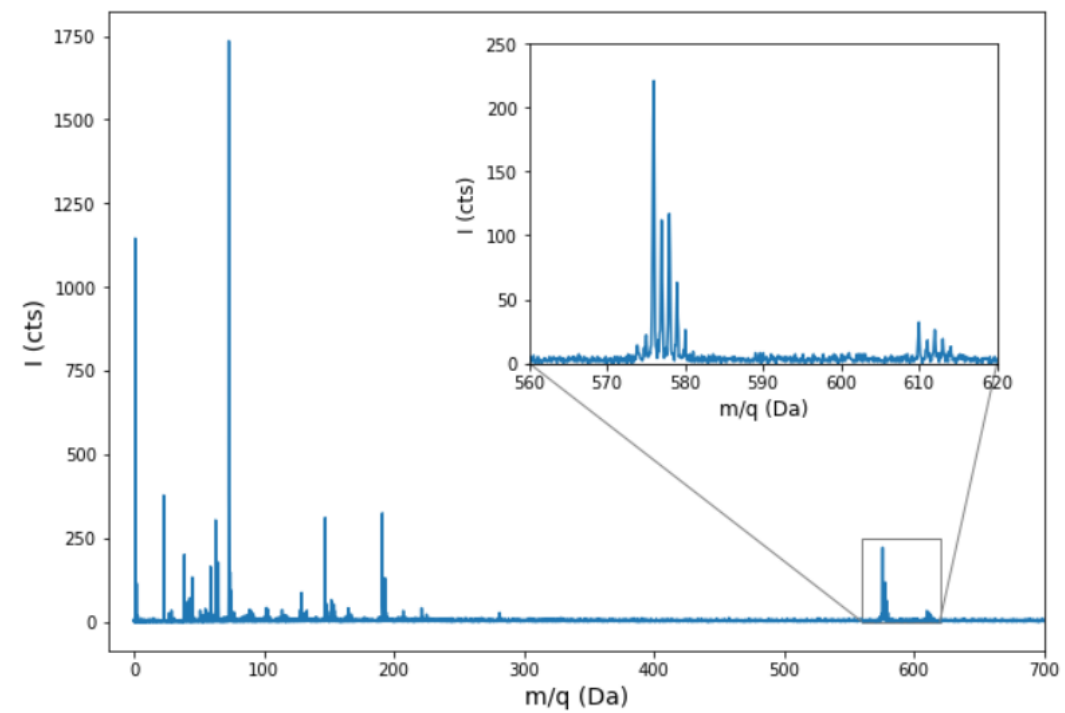

Figure 5 TOF-SIMS spectra of Phthalocyanine Blue PB15:1 using $8 \mathrm{MeV} \mathrm{Si}{ }^{4+}$ 


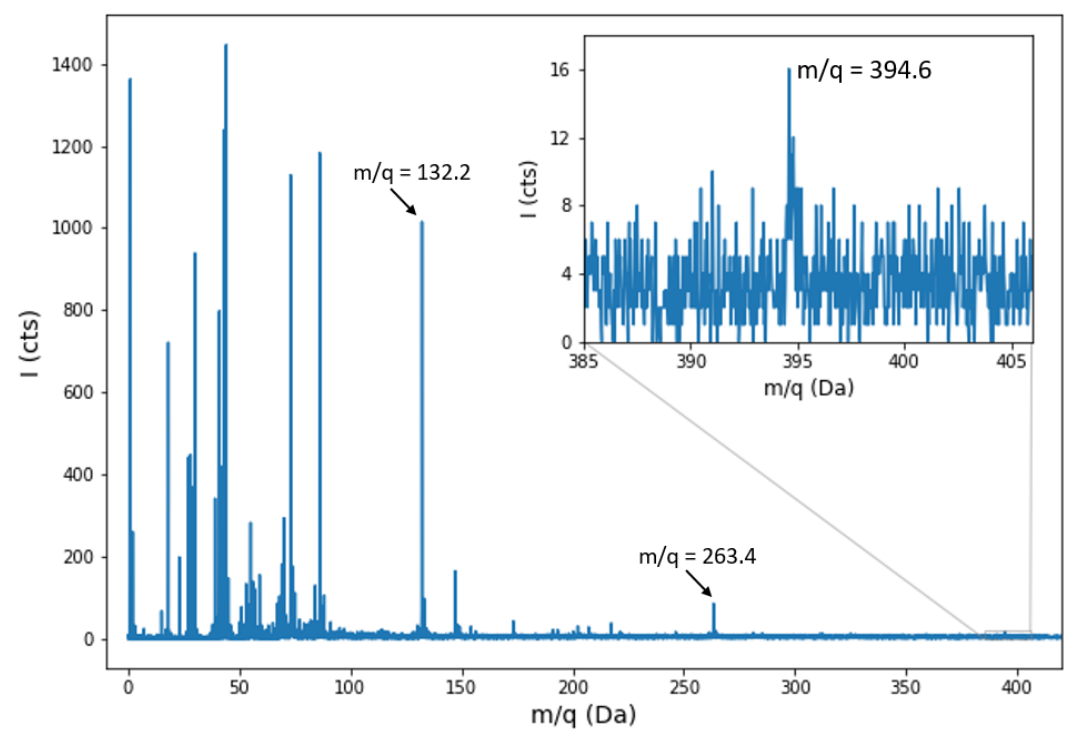

Figure 6 TOF-SIMS spectra of protonated leucine using $8 \mathrm{MeV} \mathrm{Si}^{4+}$

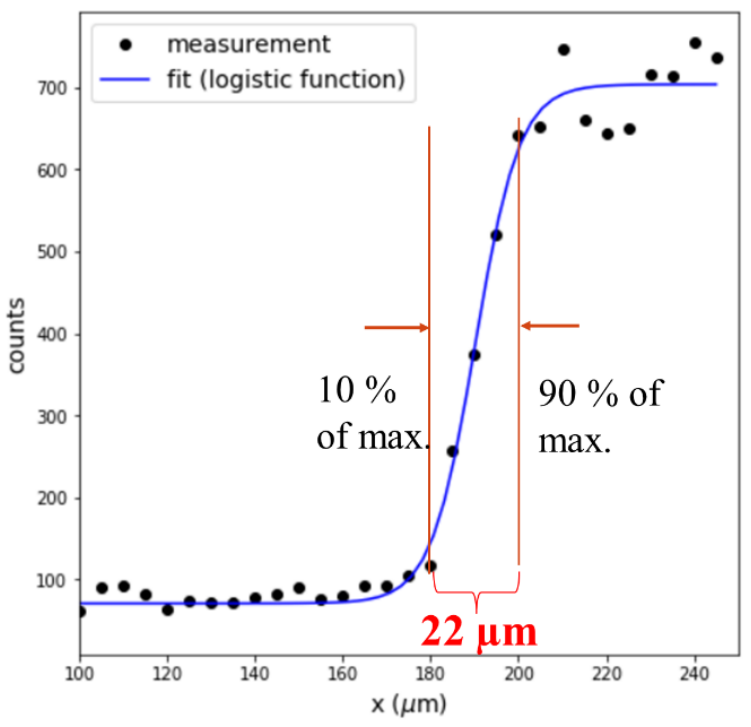

Figure 7 Resolution measurement - $250 \times 5 \mu^{2}$ line scan with $5 \mathrm{MeV} \mathrm{Si}^{4+}$ beam over the edge of leucine-vaporized $\mathrm{Si}_{3} \mathrm{~N}_{4}$ window

\begin{tabular}{|c|c|c|c|}
\hline \multicolumn{2}{|c|}{ Phthalocyanine Blue } & \multicolumn{2}{c|}{ Leucine } \\
\hline$m / q(D a)$ & $m / F W H M(m)$ & $m / q(D a)$ & $m / F W H M(m)$ \\
\hline 23 & 1045 & 2 & 500 \\
\hline 129 & 1537 & 23 & 1278 \\
\hline 576 & 2482 & 132.2 & 1488 \\
\hline 610 & 2459 & 263.4 & 1458 \\
\hline
\end{tabular}

Table 1 Measured mass resolution for selected mass peaks in Phtalocyanine blue (left) and protonated leucine (right) TOF-SIMS spectra using $8 \mathrm{MeV} \mathrm{Si}^{4+}$ primary beam 